\title{
Acortamiento en la relación Tp-f/QTc tras angioplastia en pacientes con síndrome isquémico coronario agudo
}

\author{
Shortening in the Tp-e/QTC ratio after angioplasty in patients with acute coronary \\ syndrome
}

\author{
Rodolfo M. Román-Guzmán ${ }^{*}$, Jorge A. Joya-Harrison¹, Claudia A. Navarrete-López', \\ Arturo M. Cadena-Payton², Víctor Segura-Ibarra ${ }^{3}$ y Salvador B. Valdovinos-Chávez ${ }^{1}$
}

${ }^{1}$ Servicio de Medicina Interna, Hospital San José Tec Salud; ${ }^{2}$ Instituto de Cardiología, Centro Médico Zambrano Hellion, Escuela de Medicina del Tecnológico de Monterrey; ${ }^{3}$ Escuela de Ingeniería y Ciencias, Tecnológico de Monterrey. Monterrey, Nuevo León, México

\begin{abstract}
Resumen
Objetivo: La dispersión transmural de la repolarización ventricular (DTMRV) es un factor de riesgo para muerte en pacientes con síndrome isquémico coronario agudo (SICA). Con el objetivo de conocer el efecto de la realización de angioplastia sobre la DTMRV, se estudió la relación Tp-f/QTC en pacientes con SICA sometidos a angioplastia. Método: Se diseñó un estudio observacional, retrospectivo y descriptivo. Se incluyeron 150 pacientes $(N=150)$ con diagnóstico de SICA. Se valoró la relación Tp-f/QTc inicial y se evaluó su acortamiento posangioplastia. Como objetivo secundario, se comparó la asociación de dichos cambios en la relación Tp-f/QTc con la mortalidad cardiovascular y los eventos adversos cardiovasculares. Resultados: El promedio en la relación Tp-f/QTc inicial fue de 0.2529, mientras que posangioplastia fue de 0.2397. Por medio de prueba de rangos de Wilcoxon se evidenció un descenso significativo en la relación Tp-f/QTc posterior a la angioplastia, con un valor $Z$ de -2.051 y una $p<0.04$. En el análisis secundario se encontró que una $T p-f / Q T c \geq 0.29$ posangioplastia es factor de riesgo para presentación de los siguientes eventos adversos: muerte intrahospitalaria (7.4 vs 0\%; $p<0.003)$, nuevo SICA en seguimiento a 1 año (25.9 vs. 18.5\%; $p<0.006)$ y reintervención en seguimiento a 1 año (29.6 vs. $15.0 \% ; p<0.002)$. Conclusiones: Existe un acortamiento significativo en la relación Tp-f/QTc posangioplastia en pacientes con SICA. Esta medida de la DTMRV puede servir como un predictor de muerte intrahospitalaria, eventos cardiovasculares y reintervención a 1 año en pacientes con SICA tratados con angioplastia.
\end{abstract}

Palabras clave: DTMRV. SICA. Tp-f. Tp-f/QT.

\begin{abstract}
Objective: Transmural Dispersion of Repolarization (TDR) is a Risk factor for Death in patients with Acute Coronary Syndrome (ACS). In order to know the effect of angioplasty on TDR, the Tp-e/QTC ratio was studied in patients with ACS undergoing angioplasty. Method: An observational, retrospective and descriptive study was designed. 150 patients $(N=150)$ with diagnosis of ACS were included. The initial Tp-e/QTc ratio was assessed and then its post-angioplasty shortening was evaluated.

\section{Correspondencia:}

*Rodolfo M. Román-Guzmán

E-mail: rodolfo.roman.guzman @ gmail.com

Disponible en internet: 23-12-2020 Arch Cardiol Mex. 2020;90(4):452-466 www.archivoscardiologia.com 1405-9940 / @ 2020 Instituto Nacional de Cardiología Ignacio Chávez. Publicado por Permanyer. Este es un artículo open access bajo la licencia CC BY-NC-ND (http://creativecommons.org/licenses/by-nc-nd/4.0/).
\end{abstract}


As a secondary objective, we compared the association of these Tp-e/QTC ratio changes with cardiovascular mortality and cardiovascular adverse events. Results: The average in the initial Tp-e/QTc ratio was 0.2529 , while post-angioplasty was 0.2397. Through a Wilcoxon rage test, a significant decrease in the Tp-e/QTc ratio after angioplasty was observed, with a $Z$ value of -2.051 and $p<0.04$. In the secondary analysis, it was found that a Tp-e/QTc $\geq 0.29$ post-angioplasty is a risk factor for presenting the following adverse events: in-hospital death (7.4 vs. $0 \% ; p<0.003)$, new ACS in 1-year follow-up (25.9 vs. $18.5 \% ; p<0.006)$, and reintervention in 1 -year follow up (29.6 vs. $15 \% ; p<0.002)$. Conclusions: There is a significant shortening in the Tp-e/QTC ratio post-angioplasty in patients with ACS. This measure of TDR can serve as a predictor of in-hospital death, cardiovascular events and 1-year reintervention in patients with ACS treated initially by angioplasty.

Key words: ACS. TDR. Tp-e. Tp-f/QT.

\section{Introducción}

De acuerdo con las cifras del Instituto Nacional de Estadística y Geografía, en 2016, las enfermedades del corazón fueron la primera causa de muerte en México. El $71 \%$ tuvieron como origen la cardiopatía isquémica, lo que representa el $14 \%$ del total de fallecimientos en nuestro país'.

La causa principal de muerte súbita cardiaca en pacientes con cardiopatía isquémica son las arritmias ventriculares malignas. De acuerdo con el estudio de Bayés de Luna, et al. ${ }^{2}$, aproximadamente el $80 \%$ de los casos de muerte súbita cardiaca son producto de una taquiarritmia, ya sea taquicardia ventricular o fibrilación ventricular, y solo el $20 \%$ se deben a bradiarritmias como ritmos idioventriculares, bloqueos auriculoventriculares 0 asistolia. Se han propuesto diferentes parámetros como predictores de arritmias ventriculares malignas, pero ninguno por sí solo ha demostrado tener las suficientes sensibilidad y especificidad para pronosticar este devastador evento.

Desde la década de 1990 se prestó atención a la dispersión transmural de la repolarización ventricular (DTMRV) como factor de riesgo para muerte súbita cardiaca, con principal enfoque en el intervalo QTC. Más recientemente se ha estudiado el intervalo que comprende del pico al final de la onda $T$ (Tp-f) y la relación Tp-f/QTc como índices de DTMRV.

La onda T comienza cuando la meseta del potencial de acción epicárdico se dispersa de las células mediomiocárdicas (región M). Mientras el epicardio inicia su repolarización, el gradiente de voltaje entre el epicardio y la región $\mathrm{M}$ genera el extremo ascendente de la onda $\mathrm{T}$. El gradiente de voltaje entre la región $\mathrm{M}$ y el epicardio alcanza un pico cuando el epicardio está completamente repolarizado; esto marca el pico de la onda T. En el otro extremo de la pared ventricular, la meseta endocárdica se aleja de la de las células $\mathrm{M}$, generando un gradiente de voltaje opuesto y la corriente correspondiente que limita la amplitud de la onda $\mathrm{T}$ y contribuye a la parte inicial de su extremo descendente. Así pues, el gradiente de voltaje entre el endocardio y la región $\mathrm{M}$ alcanza un pico cuando el endocardio está completamente repolarizado. Posteriormente, el gradiente continúa declinando mientras las células M concluyen su repolarización. El final de la onda $\mathrm{T}$, de esta forma, representa la extinción del gradiente cuando las células $M$ están por completo repolarizadas. Por lo tanto, podemos decir que el potencial de acción de las células $\mathrm{M}$ determina el intervalo $\mathrm{QT}^{3}$ (Fig. 1).

Cuando la onda $T$ es positiva, la región epicárdica es la que primero se repolariza, y las células $M$ serán las últimas en hacerlo. La repolarización completa del potencial epicárdico coincide con el pico de la onda T, y el final de la repolarización de las células $M$ con su final. Este intervalo entre el pico de la onda $T$ y su final se conoce como intervalo Tp-f, y representa un índice de DTMRV 4 .

Desde los años 1990 se prestó atención a la DTMRV como factor de riesgo para muerte súbita cardiaca. Algunos estudios han evaluado la duración del intervaIo QTc en distintas patologías, como hipertensión arterial sistémica ${ }^{5}$, miocardiopatía hipertrófica, síndrome de QT largo e inclusive infarto agudo de miocardio ${ }^{6}$. Sin embargo, de los estudios realizados por Antzelevitch $^{4}$ surgió la conclusión de que el Tp-f constituye un reflejo más fiel de la DTMRV, y por lo tanto un mejor predictor del sustrato arritmogénico.

A día de hoy, no obstante, se sabe que a medida que el peso corporal aumenta se produce un incremento lineal del intervalo QT que se acompaña de un aumento paralelo del Tp-f. Por si fuera poco, a medida que la frecuencia cardiaca aumenta de 60 a 100 latidos por minuto, el Tp-f disminuye de forma lineal de 40 hasta $100 \mathrm{~ms}^{7}$. Sin embargo, se ha estudiado la relación entre estos dos intervalos electrocardiográficos y se ha llegado a la conclusión de que la relación Tp-f/QT permanece relativamente constante con el mínimo rango de 0.15 a 0.25 , lo que lo hace una medida electrocardiográfica potencialmente premonitoria para estratificar el 


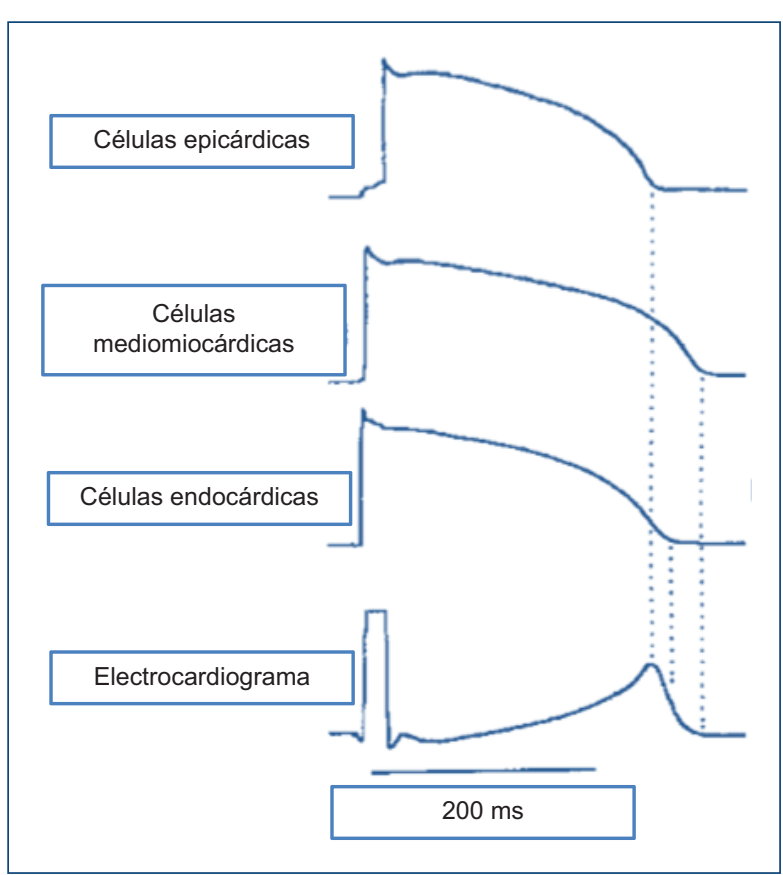

Figura 1. Representación gráfica de los perfiles electrofisiológicos de las células epicárdicas, mediomiocárdicas y endocárdicas.

riesgo de arritmias ventriculares malignas 0 de sus recidivas ${ }^{8}$.

En un estudio publicado por Erikssen, et al. ${ }^{9}$ en 2012 se evaluó la parte terminal del intervalo QT, es decir, el intervalo Tp-f, como predictor de mortalidad posterior a un infarto agudo de miocardio. Estos autores evaluaron de forma prospectiva el intervalo Tp-f en 1359/1385 (98.2\%) pacientes consecutivos con infarto agudo de miocardio con elevación del segmento ST (IAMCEST) $(n=525)$ o sin elevación del segmento ST (IAMSEST) $(n=859)$ admitidos a una unidad coronaria del Hospital Universitario de Oslo, en Noruega. Encontraron una correlación significativa entre la duración del Tp-f y la edad, la frecuencia cardiaca, la fracción de eyección del ventrículo izquierdo, la creatinina, la enfermedad coronaria trivascular, el antecedente de infarto agudo de miocardio y la duración del QRS y del QT. En el periodo de seguimiento de 1.3 años, 109 (7.9\%) pacientes fallecieron, 25 de ellos por arritmia, 45 por muerte cardiaca no relacionada con arritmia y 39 por otras causas no cardiacas. La derivación V2 fue la que mostró el intervalo Tp-f de mayor duración en el $86 \%$ de los casos. El Tp-f largo fue un fuerte predictor de riesgo de muerte y prevaleció como tal posterior a la realización de un análisis multivariado de Cox, con un riesgo relativo (RR) de 1.5 (intervalo de confianza del 95\% [IC
95\%]: 1.3-1.7). El Tp-f fue particularmente un buen predictor de riesgo de arritmias ventriculares malignas, con un RR de 1.6 (IC 95\%: 1.2-2.1). Los hallazgos fueron similares en el IAMCEST y en el IAMSEST.

De igual forma, en 2014, el mismo grupo de la Universidad de Oslo ${ }^{10}$ publicó un estudio prospectivo que evaluó 76 pacientes con antecedente de infarto agudo de miocardio que fueron sometidos a implantación de un desfibrilador automático implantable. Se registraron el electrocardiograma (ECG) basal y los eventos en el periodo de seguimiento, y se evaluaron los episodios de arritmias ventriculares que requirieron terapia antitaquicardia o descarga de choque por parte del desfibrilador. En un periodo de seguimiento de 3.5 años, el $47 \%$ de los pacientes presentaron arritmias. El intervalo Tp-f fue más largo en los pacientes que registraron arritmias ventriculares que en aquellos que no las presentaron (116 \pm 26 vs. $102 \pm 20 \mathrm{~ms} ; \mathrm{p}=0.01$ ).

En 2012, Zhao, et al..$^{11}$ publicaron un estudio de 338 pacientes con IAMCEST que valoraba la asociación entre la relación Tp-f/QT y el pronóstico en pacientes sometidos a intervención coronaria percutánea. Estos investigadores encontró que la relación Tp-f/QT guardaba una correlación con el pronóstico tanto a corto como a largo plazo. De los 388 pacientes incluidos en el estudio, 115 (34\%) tuvieron una relación Tp-f/ QT $\geq 0.29$. Este grupo de pacientes con Tp-f/ QT $\geq 0.29$ mostraron una mayor mortalidad intrahospitalaria ( 21.9 vs. $15.3 \%$; $p<0.001$ ) y un mayor número de eventos adversos cardiovasculares (48.1 vs. $15.3 \%$; $p<0.005)$. Posterior al alta, la relación Tp-f/QT $\geq 0.29$ prevaleció como predictor de mortalidad por cualquier causa ( 35.5 vs. $5.2 \%$; $p$ 0.001) y de muerte cardiaca (32.3 vs. $2.6 \% ; p<0.001)$.

Este artículo tiene como objetivo estudiar el comportamiento de la DTMRV en pacientes con síndrome isquémico coronario agudo (SICA) posangioplastia, siendo el primero de su clase en desarrollarse en México.

\section{Método}

Con el objetivo de conocer el efecto de la realización de angioplastia sobre la relación Tp-f/QTc en pacientes con SICA, se diseñó un estudio observacional, retrospectivo, con un análisis descriptivo de la asociación de dichos cambios electrocardiográficos y la presentación de eventos adversos. Se incluyeron 150 pacientes mayores de 18 años $(n=150)$ que acudieron con diagnóstico de SICA sometidos a angioplastia en los hospitales San José y Zambrano Hellion Tec Salud, en el periodo 
comprendido del 1 de enero de 2014 al 31 de diciembre de 2017, valorando los intervalos QTc y Tp-f , y la relación Tp-f/QTc en su ECG inicial.

Se evaluó el acortamiento posangioplastia de la relación Tp-f/QTc y su asociación con la mortalidad y la presentación de eventos adversos durante la estancia intrahospitalaria y en el seguimiento a 1 año. Se consideró la morbimortalidad intrahospitalaria por los siguientes eventos: choque cardiogénico, arritmia intrahospitalaria, insufiencia cardiaca aguda, muerte intrahospitalaria, arritmias ventriculares malignas (tanto fibrilación ventricular como taquicardia ventricular) y asistolia que requiriera reanimación cardiopulmonar. En el seguimiento a 1 año se valoraron la reintervención, un nuevo SICA y la muerte por cualquier causa.

Se estandarizó que los ECG considerados para su análisis fuesen de 12 derivaciones y obtenidos a $25 \mathrm{~mm} / \mathrm{s}$. El ECG preangioplastia valorado en cada caso correspondió al que se realizó previamente a la angioplastia. En los casos en que la intervención fue primaria, correspondió al ECG obtenido en el momento del arribo del paciente a la sala de urgencias. En los casos en que se realizó angioplastia temprana, se consideró el último ECG obtenido antes de la angioplastia. El ECG posangioplastia considerado para el análisis tras la intervención correspondió al ECG obtenido el día inmediato posterior a la angioplastia.

El investigador encargado de la revisión de los ECG recibió entrenamiento bajo supervisión de un cardiólogo especialista en electrofisiología antes de su participación en el estudio. En los pacientes con IAMCEST, el Tp-f se evaluó en las derivaciones correspondientes al territorio del vaso afectado, considerando para la medición aquella derivación con mayor elevación del punto J.

En los pacientes con IAMSEST y angina inestable se valoró la derivación V2 o V5, según criterio apreciativo del investigador. El método empleado en la medición del Tp-f fue el «método de la cola», que consiste en la medición del intervalo del pico máximo de la onda $T$ hasta el punto en que la pendiente de la rama descendente de la onda T cruza la línea de base isoeléctrica. El intervalo QT se midió desde el inicio del complejo QRS hasta el punto en que la tangente de la máxima pendiente de la rama descendente de la onda T cruza la línea de base isoeléctrica. Posteriormente, de forma consistente con otros grupos de investigación, se hizo una corrección para obtener el QTc según la fórmula de Bazzet: QTc $=\mathrm{QT} \sqrt{ }(\mathrm{RR})$. Por último, se realizó un cálculo fraccional para obtener el valor definitivo de la relación Tp-f/QTc.
Para la obtención de los datos se utilizaron los expedientes clínicos de los hospitales (Laserfiche ${ }^{\circledR}$ ) y de consulta externa (Alert ${ }^{\circledR}$ e ISSSTELEON). Para el análisis estadístico se utilizó el sistema SPSS Statistics ${ }^{\circledR}$ Versión 25, Microsoft ${ }^{\circledR}$ Excel $^{\circledR}$ for 2011 Versión 14.4.2 (14509) y Microsoft ${ }^{\circledR}$ Power Point ${ }^{\circledR}$ for Mac 2011 Versión 14.4.2 (140509).

Se emplearon medias y desviaciones estándar para variables cuantitativas continuas y discretas, y para variables categóricas se emplearon frecuencias o porcentajes. Para las variables cuantitativas de distribución anormal se emplearon la mediana y los rangos intercuartílicos.

Se utilizaron la prueba de ji al cuadrado para las variables cualitativas y la prueba t de Student (en casos de distribución normal) o la prueba de rangos de Wilcoxon (en casos de distribución anormal) para las variables cuantitativas. Se determinó un IC 95\%, con un valor de $p \leq 0.05$ en dos direcciones para valores estadísticamente significativos.

\section{Resultados}

Tras la revisión de los Censos de Cardiología del Sistema Tec Salud se obtuvieron 393 episodios elegibles, correspondientes a pacientes registrados por cuadro de dolor torácico que acudieron a las unidades de emergencias de los hospitales San José y Zambrano Hellion Tec Salud, en el periodo comprendido del 1 de enero de 2014 al 31 de diciembre de 2017. Siguiendo los criterios de inclusión y de exclusión se descartaron 243 episodios, resultando en un total de 150 pacientes. En la figura 2 se muestra un diagrama de flujo que ejemplifica el proceso de selección y exclusión de la fase 2 de recolección de pacientes y sus ECG.

En la muestra estudiada (Tabla 1), la edad promedio fue de $61.6 \pm 11.44$ años. El $90.7 \%$ de los pacientes eran hombres. Las afecciones concomitantes que más prevalecieron fueron las siguientes: hipertensión arterial, presente en el $46.7 \%$ de los participantes; tabaquismo, en el $40.7 \%$; diabetes mellitus tipo 2, en el $34 \%$; y dislipidemia, en el $30 \%$. Solo el $23.3 \%$ de los pacientes tenían antecedentes de cardiopatía isquémica, mientras que un mínimo porcentaje (4\%) tenían antecedentes de insuficiencia cardiaca.

El $100 \%$ de los pacientes estudiados recibieron angioplastia coronaria durante su internamiento, pero solo en el $26.6 \%$ de ellos se hizo angioplastia urgente. De estos casos, el mínimo tiempo puerta-aguja fue de 5 minutos, con un máximo de 147 minutos y una media de $50.38 \pm 32.5$ minutos. Por otra parte, 


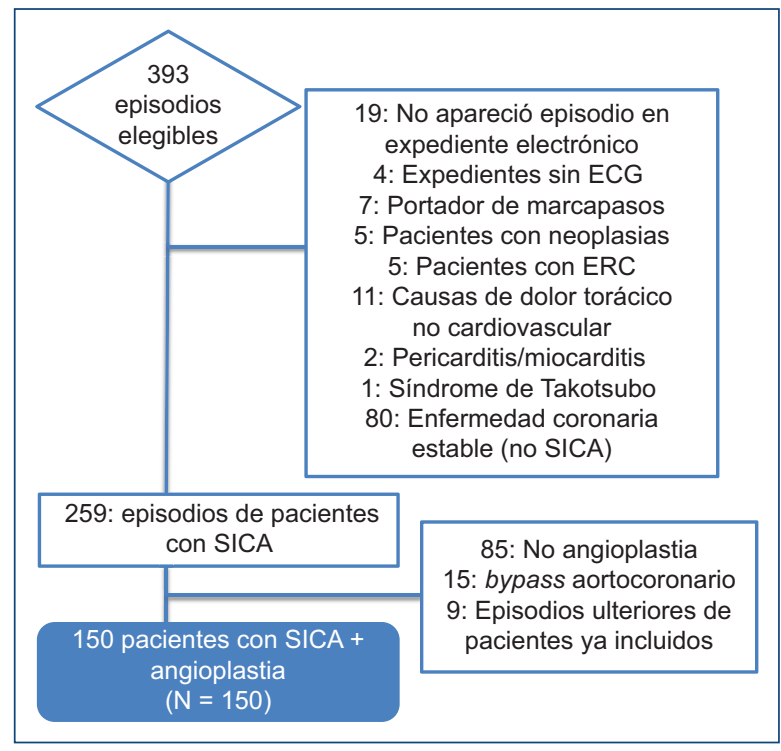

Figura 2. Selección de pacientes basada en criterios de inclusión y exclusión. ECG: electrocardiograma; ERC: Enfermedad renal crónica; SICA: síndrome isquémico coronario agudo.

Tabla 1. Características demográficas, tipo de síndrome isquémico coronario agudo y vaso afectado

\begin{tabular}{|l|c|c|}
\hline Característica & \multicolumn{2}{|c|}{ Frecuencia observada } \\
\hline Edad & \multicolumn{2}{|c|}{$61.6 \pm 11.44$} \\
\hline & $\mathbf{n}$ & $\%$ \\
\hline Sexo & & \\
Masculino & 136 & 90.7 \\
Femenino & 14 & 9.3 \\
\hline Comorbilidad & & \\
HAS & 70 & 46.7 \\
DM2 & 51 & 34 \\
Dislipidemia & 45 & 30 \\
\hline Tabaquismo & 61 & 40.7 \\
Cardiopatía isquémica & 35 & 23.3 \\
Insuficiencia cardiaca & 6 & 4 \\
\hline Tipo de SICA & & \\
Angina inestable & 18 & 12 \\
\hline IAMSES & 59 & 39.3 \\
IAMCEST & 73 & 48.7 \\
\hline Vaso afectado & & \\
CD & 41 & 27.3 \\
DA & 61 & 40.7 \\
Cx & 16 & 10.7 \\
DA + CD & 10 & 6.7 \\
Cx + DA & 8 & 5.3 \\
Cx + CD & 9 & 6 \\
Enfermedad trivascular & 5 & 3.3 \\
\hline
\end{tabular}

CD: coronaria derecha; $\mathrm{Cx}$ : circunfleja; DA: descendente anterior; DM2: diabetes mellitus tipo 2; HAS: hipertensión arterial sistémica; IAMCEST: infarto agudo de miocardio con elevación del segmento ST; IAMSEST: infarto agudo de miocardio con elevación del segmento ST; SICA: síndrome isquémico coronario agudo. el tiempo mínimo puerta-angioplastia fue de 14 minutos, con un máximo de 172 minutos y una media de $71.4 \pm 35.27$ minutos.

El $48.7 \%$ de los casos correspondieron a IAMCEST, el $39.3 \%$ fueron IAMSEST y hubo un $12 \%$ de anginas inestables. Con un $40.7 \%$ de los casos, la arteria descendente anterior fue el vaso mayormente afectado, seguido de la coronaria derecha con el $27.3 \%$; en el $18 \%$ de los casos se vio afectado más de un vaso.

El promedio de la relación Tp-f/QTc en el ECG inicial fue de $0.2529 \pm 0.0697$, mientras que en el ECG posangioplastia fue de $0.2397 \pm 0.07200$.

Se realizó la prueba de Kolgomorov-Smirnov para valorar la distribución del acortamiento de la relación Tp-f/QTc y se encontró un valor $Z$ de 0.078 , con $p=0.03$, que corroboró que la distribución fue anormal, por lo cual se procedió a realizar la prueba de rangos de Wilcoxon y se concluyó que posterior a la angioplastia se presentó un descenso significativo en la relación Tp-f/QTc, con un valor Z de -2.051 ( $p<0.04)$, con lo que se aprueba la hipótesis de que tras la realización de la angioplastia realmente se experimenta un acortamiento de la relación Tp-f/QTc.

Para el estudio de eventos adversos, tanto los ocurridos periprocedimiento como los posteriores a la intervención, se realizó un análisis univariado con prueba de ji al cuadrado, estableciendo como punto de corte una relación Tp-f/QTc $\geq 0.25$ para valorarla como medida de la DTMRV, lo cual corresponde a lo descrito previamente por Gupta, et al. ${ }^{8}$ en su estudio. Asimismo, se realizó un análisis similar con un punto de corte $\geq 0.29$, basado en los hallazgos de Zhao, et al. ${ }^{11}$ ya comentados.

En el caso de los eventos periprocedimiento, ninguno demostró guardar una relación estadísticamente significativa para una relación Tp-f/QTc $\geq 0.25$ (Tabla 2). Sin embargo, cuando se consideró una relación Tp-f/QTc $\geq 0.29$, sí se encontró una diferencia estadísticamente significativa para la presentación de eventos durante el procedimiento (22.72 vs. $9.43 \%$; $p$ $<0.029$ ). Asimismo, el tipo de arritmia presentada parece tener una relación con el grado de DTMRV (Tabla 3).

Para el análisis causa-efecto correspondiente al grado de DTMRV posterior a la angioplastia se valoró, de forma similar a los eventos preangioplastia, por medio de la prueba de ji al cuadrado, la presentación de adversos intrahospitalarios y en el seguimiento de pacientes a 1 año posterior a haber presentado el evento inicial de SICA. Se encontró que una relación Tp-f/QTc $\geq 0.25$ tuvo significación estadística por la 
Tabla 2. Eventos periprocedimiento. Análisis univariado para una relación Tp-f/QTc $\geq 25$

\begin{tabular}{|c|c|c|c|c|}
\hline Variable & $\begin{array}{c}\text { Total pacientes } \\
n=150\end{array}$ & $\begin{array}{l}\text { Tp-f/0Tc } \geq 0.25 \\
n=67(44.66 \%)\end{array}$ & $\begin{array}{l}\text { Tp-f/OTc }<0.25 \\
n=83(55.33 \%)\end{array}$ & $\mathbf{p}$ \\
\hline Arritmia & $22(14.6)$ & $12(17.9)$ & $10(11.76)$ & 0.313 \\
\hline Arritmia que ameritó $\mathrm{RCP}$ & $6(4)$ & $3(4.4)$ & $3(3.6)$ & 0.789 \\
\hline Muerte durante el procedimiento & $3(2)$ & $2(2.9)$ & $1(1.2)$ & 0.439 \\
\hline Evento durante el procedimiento & $20(13.3)$ & $12(17.9)$ & $8(9.6)$ & 0.138 \\
\hline $\begin{array}{l}\text { Tipo de arritmia } \\
\text { Fibrilación auricular } \\
\text { Fibrilación ventricular } \\
\text { Taquicardia ventricular } \\
\text { Taquicardia supraventricular } \\
\text { Asistolia } \\
\text { Bradiarritmia }\end{array}$ & $\begin{array}{c}4(2.6) \\
4(2.6) \\
2(1.3) \\
1(0.6) \\
3(2) \\
6(4)\end{array}$ & $\begin{array}{c}1(1.4) \\
3(4.4) \\
2(2.9) \\
0(0) \\
1(1.4) \\
5(7.4)\end{array}$ & $\begin{array}{c}3(3.6) \\
1(1.2) \\
0(0) \\
1(1.2) \\
2(2.4) \\
1(1.2)\end{array}$ & 0.148 \\
\hline
\end{tabular}

RCP: reanimación cardiopulmonar.

Tabla 3. Eventos periprocedimiento. Análisis univariado para una relación Tp-f/QTc $\geq 0.29$

\begin{tabular}{|c|c|c|c|c|}
\hline Variable & $\begin{array}{c}\text { Total pacientes } \\
n=150\end{array}$ & $\begin{array}{l}\text { Tp-f/0Tc } \geq 0.29 \\
n=44(29.33 \%)\end{array}$ & $\begin{array}{c}\text { Tp-f/0Tc }<0.29 \\
n=106(70.66 \%)\end{array}$ & $\mathbf{p}$ \\
\hline Arritmia & $22(14.6)$ & $10(22.7)$ & $12(11.3)$ & 0.720 \\
\hline Arritmia que ameritó $\mathrm{RCP}$ & $6(4)$ & $3(6.8)$ & $3(2.8)$ & 0.256 \\
\hline Muerte durante el procedimiento & $3(2)$ & $2(4.5)$ & $1(0.9)$ & 0.151 \\
\hline Evento durante el procedimiento & $20(13.3)$ & $10(22.7)$ & $10(9.4)$ & 0.029 \\
\hline $\begin{array}{l}\text { Tipo de arritmia } \\
\text { Fibrilación auricular } \\
\text { Fibrilación ventricular } \\
\text { Taquicardia ventricular } \\
\text { Taquicardia supraventricular } \\
\text { Asistolia } \\
\text { Bradiarritmia }\end{array}$ & $\begin{array}{c}4(2.6) \\
4(2.6) \\
2(1.3) \\
1(0.6) \\
3(2) \\
6(4)\end{array}$ & $\begin{array}{c}0(0) \\
2(4.5) \\
2(45) \\
0(0) \\
1(2.2 \\
5(11.3)\end{array}$ & $\begin{array}{l}4(3.7) \\
2(1.8) \\
2(1.8) \\
1(0.9) \\
2(1.8) \\
1(0.9)\end{array}$ & 0.010 \\
\hline
\end{tabular}

RCP: reanimación cardiopulmonar.

prueba de ji al cuadrado para reintervención por SICA en el seguimiento a 1 año (25.9 vs. $12.9 \%$; $p<0.019$, y asimismo para la presentación de cualquier evento cardiovascular a 1 año (31.4 vs. $16.1 \%$; $p<0.030$. Al establecer un punto de corte en la relación Tp-f/QTc $\geq$ 0.29 se encontró significación estadística para la presentación de los siguientes efectos adversos: muerte intrahospitalaria (7.4 vs. $0 \% ; p<0.003$ ), nuevo SICA en el seguimiento a 1 año ( 25.9 vs. $18.5 \%$; $p<0.006)$, reintervención ( 29.6 vs. $15.0 \% ; p<0.002$ ) y presentación de cualquier evento cardiovascular a 1 año (37 vs. $18.3 \% ; p<0.033)$. Estos resultados quedan resumidos en las tablas 4 y 5 .

Basándose en los hallazgos previamente mencionados, se analizó por medio de regresión logística para el análisis de los eventos que resultaron significativos en el análisis univariado. Sin embargo, no se encontró una significación estadística en ninguno de los eventos clínicos. Estos resultados, no obstante, pueden guardar relación con el carácter retrospectivo del estudio, ya que no se diseñó primariamente un estudio para valoración de la mortalidad.

Se decidió analizar por medio de curvas ROC (Receiver Operating Characteristic) la sensibilidad y la especificidad de la relación Tp-f/QTc como prueba predictiva para la presentación de ciertos eventos. De este análisis resultó que, al valorar la mortalidad intrahospitalaria por medio de curva ROC para la relación Tp-f/QTc preangioplastia, el área bajo la curva resultó ser de 0.766 , con $p<0.044$ (Fig. 3). Por otro lado, la relación Tp-f/QTc posangioplastia mostró un área bajo la curva de 0.952 , con $p<0.028$, para la predicción de 
Tabla 4. Eventos intrahospitalarios y a 1 año del evento de síndrome isquémico coronario agudo primario. Análisis univariado para $\mathrm{Tp}-\mathrm{f} / \mathrm{QTc} \geq 0.25$

\begin{tabular}{|c|c|c|c|c|}
\hline Variable & $\begin{array}{c}\text { Total pacientes } \\
n=147\end{array}$ & $\begin{array}{l}\text { Tp-f/0Tc } \geq 0.25 \\
n=54(36.73 \%)\end{array}$ & $\begin{array}{l}\text { Tp-f/0Tc }<0.25 \\
n=93(63.26 \%)\end{array}$ & $\mathbf{p}$ \\
\hline Insuficiencia cardiaca aguda & $17(11.5)$ & $6(11.1)$ & $11(11.8)$ & 0.896 \\
\hline Choque cardiogénico & $10(6.8)$ & $4(7.4)$ & $6(6.4)$ & 0.824 \\
\hline Arritmia intrahospitalaria & $23(15.6)$ & $7(12.9)$ & $16(17.2)$ & 0.495 \\
\hline Muerte intrahospitalaria & $2(1.3)$ & $2(3.7)$ & $0(0)$ & 0.062 \\
\hline Evento intrahospitalario & $30(20.4)$ & $9(16.6)$ & $21(22.5)$ & 0.391 \\
\hline Evento cardiovascular mayor & $6(4.0)$ & $2(3.7)$ & $4(4.3)$ & 0.860 \\
\hline $\begin{array}{l}\text { Tipo de arritmia } \\
\text { Fibrilación auricular } \\
\text { Fibrilación ventricular } \\
\text { Taquicardia ventricular } \\
\text { Taquicardia supraventricular } \\
\text { Asistolia } \\
\text { Bradiarritmia }\end{array}$ & $\begin{array}{c}7(4.7) \\
0(0) \\
3(2.0) \\
2(1.3) \\
0(0) \\
11(7.4)\end{array}$ & $\begin{array}{c}0(0) \\
0(0) \\
1(1.8) \\
0(0) \\
0(0) \\
5(9.2)\end{array}$ & $\begin{array}{c}7(7.5) \\
0(0) \\
2(2.1) \\
2(2.1) \\
0(0) \\
6(6.4)\end{array}$ & 0.190 \\
\hline Muerte durante el primer año & $1(0.6)$ & $0(0)$ & $1(1.0)$ & 0.131 \\
\hline Nuevo SICA & $29(19.7)$ & $14(25.9)$ & $15(16.1)$ & 0.052 \\
\hline Nueva intervención & $26(17.6)$ & $14(25.9)$ & 12 (12.9) & 0.019 \\
\hline Nuevo evento durante el primer año & $32(21.7)$ & 17 (31.4) & $15(16.1)$ & 0.030 \\
\hline
\end{tabular}

SICA: síndrome isquémico coronario agudo.

Tabla 5. Eventos intrahospitalarios y a 1 año del evento de síndrome isquémico coronario agudo primario. Análisis univariado para $\mathrm{Tp}-\mathrm{f} / \mathrm{QTc} \geq 0.25$

\begin{tabular}{|c|c|c|c|c|}
\hline Variable & $\begin{array}{l}\text { Total pacientes } \\
\qquad n=147\end{array}$ & $\begin{array}{l}\text { Tp-f/QTc } \geq 0.29 \\
n=27(18.37 \%)\end{array}$ & $\begin{array}{c}\text { Tp-f/QTc }<0.29 \\
n=120(81.63 \%)\end{array}$ & $\mathbf{p}$ \\
\hline Insuficiencia cardiaca aguda & $17(11.5)$ & $4(14.8)$ & $13(10.8)$ & 0.559 \\
\hline Choque cardiogénico & $10(6.8)$ & $2(7.4)$ & $8(6.6)$ & 0.890 \\
\hline Arritmia intrahospitalaria & $23(15.6)$ & $3(11.1)$ & $20(16.6)$ & 0.473 \\
\hline Muerte intrahospitalaria & $2(1.3)$ & $2(7.4)$ & $0(0)$ & 0.003 \\
\hline Evento intrahospitalario & $30(20.4)$ & $4(14.8)$ & $26(21.6)$ & 0.425 \\
\hline Evento cardiovascular mayor & $6(4.0)$ & $1(3.7)$ & $5(4.1)$ & 0.913 \\
\hline Muerte durante el primer año & $1(0.6)$ & $0(0)$ & $1(0.8)$ & 0.010 \\
\hline Nuevo SICA & $29(19.7)$ & $7(25.9)$ & $22(18.33)$ & 0.006 \\
\hline Nueva intervención & $26(17.6)$ & $8(29.6)$ & $19(15)$ & 0.002 \\
\hline Nuevo evento durante el primer año & $32(21.7)$ & $10(37.0)$ & $22(18.3)$ & 0.033 \\
\hline $\begin{array}{l}\text { Tipo de arritmia } \\
\text { Fibrilación auricular } \\
\text { Fibrilación ventricular } \\
\text { Taquicardia ventricular } \\
\text { Taquicardia supraventricular } \\
\text { Asistolia } \\
\text { Bradiarritmia }\end{array}$ & $\begin{array}{c}7(4.7) \\
0(0) \\
3(2.0) \\
2(1.3) \\
0(0) \\
11(7.4)\end{array}$ & $\begin{array}{c}0(0) \\
0(0) \\
1(3.7) \\
0(0) \\
0(0) \\
2(7)\end{array}$ & $\begin{array}{c}7(5.8) \\
0(0) \\
2(1.6) \\
2(1.6) \\
0(0) \\
9(7.5)\end{array}$ & 0.730 \\
\hline
\end{tabular}

SICA: síndrome isquémico coronario agudo. 
ROC MedidaRelTpfQTpreBY MuerteIntrahospitalaria(1)

/PLOT=CURVE(REFERENCE)

/PRINT=SE COORDINATES

/CRITERIA=CUTOFF(INCLUDE) TESTPOS(LARGE) DISTRIBUTION(FREE) CI (95)

/MISSING=EXCLUDE.

\section{Curva COR}

\section{Notas}

\begin{tabular}{|c|c|c|}
\hline \multicolumn{2}{|l|}{ Salida creada } & 27-SEP-2019 14:23:47 \\
\hline \multicolumn{3}{|l|}{ Comentarios } \\
\hline \multirow[t]{6}{*}{ Entrada } & Datos & $\begin{array}{l}\text { IUsers/rodolforoman/Dr } \\
\text { opbox/DOCUMENTOS } \\
\text { TESIS/BASE DE DATOS } \\
\text { TESIS RODOLFO } \\
\text { ROMAN_01.sav }\end{array}$ \\
\hline & Conjunto de datos activo & ConjuntoDatos1 \\
\hline & Filtro & <ninguno > \\
\hline & Ponderación & $<$ ninguno $>$ \\
\hline & Segmentar archivo & <ninguno > \\
\hline & $\begin{array}{l}\mathrm{N} \text { de filas en el archivo } \\
\text { de datos de trabajo }\end{array}$ & 150 \\
\hline \multirow[t]{2}{*}{$\begin{array}{l}\text { Gestión de valores } \\
\text { perdidos }\end{array}$} & Definición de perdidos & $\begin{array}{l}\text { Los valores perdidos } \\
\text { definidos por el usuario } \\
\text { se tratan como } \\
\text { perdidos. }\end{array}$ \\
\hline & Casos utilizados & $\begin{array}{l}\text { Las estadísticas se } \\
\text { basan en todos los } \\
\text { casos con datos válidos } \\
\text { para todas las variables } \\
\text { del análisis. }\end{array}$ \\
\hline Sintaxis & & $\begin{array}{l}\text { ROC } \\
\text { MedidaRelTpfQTpre BY } \\
\text { Muertelntrahospitalaria } \\
\text { (1) } \\
\text { /PLOT=CURVE } \\
\text { (REFERENCE) } \\
\text { /PRINT=SE } \\
\text { COORDINATES } \\
\text { /CRITERIA=CUTOFF } \\
\text { (INCLUDE) TESTPOS } \\
\text { (LARGE) DISTRIBUTION } \\
\text { (FREE) CI(95) } \\
\text { /MISSING=EXCLUDE. }\end{array}$ \\
\hline \multirow[t]{2}{*}{ Recursos } & Tiempo de procesador & $00: 00: 00.25$ \\
\hline & Tiempo transcurrido & 00:00:00.00 \\
\hline
\end{tabular}

Figura 3. Curva ROC de la relación Tp-f/QTc preangioplastia para predicción de la mortalidad intrahospitalaria. Área bajo la curva $=0.766 ; p<0.044$ (Continúa). 


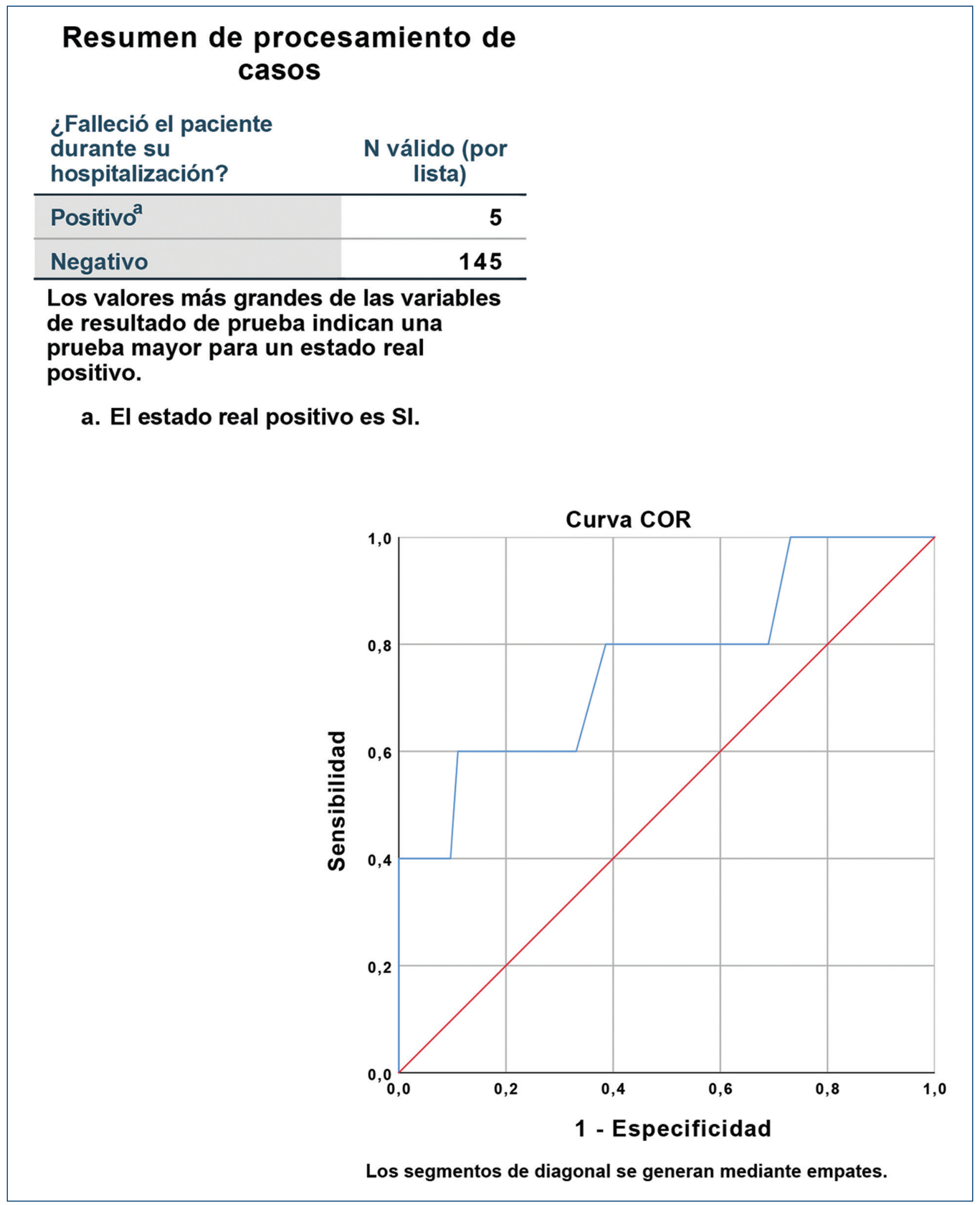

Figura 3. Curva ROC de la relación Tp-f/QTc preangioplastia para predicción de la mortalidad intrahospitalaria. Área bajo la curva $=0.766 ; p<0.044$ (Continúa). 


\section{Área bajo la curva}

Variables de resultado de prueba: ¿Cuál fue la medida de la relación Tp-f/QTc en el ECG inicial?

\begin{tabular}{c|c|c|c|c} 
& & $\begin{array}{c}\text { Significación } \\
\text { asintótica }^{n}\end{array}$ & $\begin{array}{c}\text { 95\% de intervalo de confianza } \\
\text { asintótico }\end{array}$ \\
Área & Desv. Error & & & \multicolumn{2}{c}{${ }^{\mathrm{a}}$} & & Límite inferior & Límite superior \\
\hline $\mathbf{7 6 6}$ & $\mathbf{1 2 3}$ &, $\mathbf{0 4 4}$ & $\mathbf{5 2 4}$ & $\mathbf{1 , 0 0 0}$ \\
\hline
\end{tabular}

Las variables de resultado de prueba: ¿Cuál fue la medida de la relación Tp-f/QTc en el ECG inicial? tienen, como mínimo, un empate entre el grupo de estado real positivo y el grupo de estado real negativo. Las estadísticas podrían estar sesgadas.

a. Bajo el supuesto no paramétrico

b. Hipótesis nula: área verdadera $=0,5$

\section{Coordenadas de la curva}

Variables de resultado de prueba: ¿Cuál fue la medida de la relación Tp-f/QTc en el ECG inicial?

\begin{tabular}{|c|c|c|}
\hline $\begin{array}{l}\text { Positivo si es } \\
\text { mayor o igual } \\
\text { que }\end{array}$ & Sensibilidad & $\begin{array}{c}1- \\
\text { Especificidad }\end{array}$ \\
\hline 0000 & 1,000 & 1,000 \\
\hline ,1250 & 1,000 & ,993 \\
\hline ,1350 & 1,000 & ,986 \\
\hline , 1450 & 1,000 & ,966 \\
\hline , 1550 & 1,000 & ,952 \\
\hline , 1650 & 1,000 & ,931 \\
\hline , 1750 & 1,000 & ,897 \\
\hline , 1850 & 1,000 & ,876 \\
\hline , 1950 & 1,000 & ,821 \\
\hline ,2050 & 1,000 & ,731 \\
\hline ,2150 & , 800 & ,690 \\
\hline ,2250 & 800 & ,634 \\
\hline ,2350 & , 800 &, 503 \\
\hline ,2450 & ,800 & ,448 \\
\hline ,2550 & , 800 & ,386 \\
\hline, 2650 &, 600 & ,331 \\
\hline ,2750 & ,600 & ,303 \\
\hline ,2850 & ,600 & ,283 \\
\hline ,2950 & ,600 & ,241 \\
\hline, 3050 &, 600 & ,152 \\
\hline ,3150 & ,600 & ,131 \\
\hline
\end{tabular}

Figura 3. Curva ROC de la relación Tp-f/QTc preangioplastia para predicción de la mortalidad intrahospitalaria. Área bajo la curva $=0.766 ; p<0.044$ (Continúa). 


\begin{tabular}{|c|c|c|}
\hline \multicolumn{3}{|c|}{ Coordenadas de la curva } \\
\hline \multicolumn{3}{|c|}{ Variables de resultado de prueba: ¿Cuál fue la medida de la relación Tp-f/QTc en el ECG inicial? } \\
\hline $\begin{array}{l}\text { Positivo si es } \\
\text { mayor o igual } \\
\text { que }^{\text {a }}\end{array}$ & Sensibilidad & $\begin{array}{c}1- \\
\text { Especificidad }\end{array}$ \\
\hline, 3250 &, 600 & ,124 \\
\hline ,3350 &, 600 &, 110 \\
\hline ,3450 &, 400 & 097 \\
\hline ,3550 &, 400 &, 090 \\
\hline, 3650 &, 400 &, 069 \\
\hline, 3750 &, 400 &, 055 \\
\hline ,3850 &, 400 &, 041 \\
\hline, 4000 &, 400 &, 028 \\
\hline ,4150 &, 400 &, 014 \\
\hline, 4350 &, 400 &, 007 \\
\hline, 4600 &, 400 &, 000 \\
\hline, 4850 &, 200 &, 000 \\
\hline 1,0000 &, 000 &, 000 \\
\hline \multicolumn{3}{|c|}{$\begin{array}{l}\text { Las variables de resultado de prueba: ¿Cuál } \\
\text { fue la medida de la relación Tp-f/QTc en el } \\
\text { ECG inicial? tienen, como mínimo, un empate } \\
\text { entre el grupo de estado real positivo y el } \\
\text { grupo de estado real negativo. }\end{array}$} \\
\hline \multicolumn{3}{|c|}{$\begin{array}{l}\text { a. El valor de corte más pequeño es el valor mínimo de prueba observado menos } 1 \text { y el valor de } \\
\text { corte más grande es el valor máximo de prueba observado más } 1 \text {. Todos los demás valores de } \\
\text { corte son los promedios de los dos valores de prueba observados solicitados consecutivos. }\end{array}$} \\
\hline
\end{tabular}

Figura 3. Curva ROC de la relación Tp-f/QTc preangioplastia para predicción de la mortalidad intrahospitalaria. Área bajo la curva $=0.766 ; p<0.044$.

la mortalidad intrahospitalaria (Fig. 4). No se evidenció un área bajo la curva significativa para otros eventos clínicos, ni en el caso de la relación Tp-f/QTc preanglioplastia, ni para la relación Tp-f/QTc posanglioplastia.

\section{Discusión}

Se decidió valorar el efecto de la realización de angioplastia sobre la relación Tp-f/QTc en pacientes con SICA, ya que este parámetro electrocardiográfico representa un índice de la repolarización ventricular relativamente constante, con poca influencia de otras variables. De este precepto surgió interés en nuestro grupo de investigación para valorar el efecto de una patología altamente prevalente, como lo es la cardiopatía isquémica, sobre este índice de DTMRV, y asimismo evaluar el efecto de una intervención altamente probada como modificador pronóstico, como lo es la intervención coronaria por medio de angioplastia percutánea.

Asimismo, teniendo conocimiento de la utilidad de otras medidas de DTMRV como marcadores pronósticos en pacientes con otras patologías, como el QTc y el propio Tp-f, atrajo nuestra inquietud la idea de probar la relación Tp-f/QTc como índice predictivo para la presentación de eventos cardiovasculares en pacientes con cardiopatía isquémica.

Este estudio aporta información relevante sobre una patología muy prevalente en nuestro país y sus resultados son consistentes con los de estudios de otras poblaciones. 
ROC MedidaRelTpfQTpostBY MuerteIntrahospitalaria(1)

/PLOT=CURVE(REFERENCE)

/PRINT=SE COORDINATES

/CRITERIA=CUTOFF(INCLUDE) TESTPOS(LARGE) DISTRIBUTION(FREE) CI (95)

/MISS ING=EXCLUDE.

\section{Curva COR}

\section{Notas}

\begin{tabular}{|c|c|c|}
\hline \multicolumn{2}{|l|}{ Salida creada } & 26-SEP-2019 21:13:16 \\
\hline \multicolumn{3}{|l|}{ Comentarios } \\
\hline \multirow[t]{6}{*}{ Entrada } & Datos & $\begin{array}{l}\text { IUsers/rodolforoman/Dr } \\
\text { opbox/DOCUMENTOS } \\
\text { TESIS/BASE DE DATOS } \\
\text { TESIS RODOLFO } \\
\text { ROMAN_01.sav }\end{array}$ \\
\hline & Conjunto de datos activo & ConjuntoDatos1 \\
\hline & Filtro & $<$ ninguno $>$ \\
\hline & Ponderación & <ninguno $>$ \\
\hline & Segmentar archivo & $<$ ninguno $>$ \\
\hline & $\begin{array}{l}\mathrm{N} \text { de filas en el archivo } \\
\text { de datos de trabajo }\end{array}$ & 150 \\
\hline \multirow[t]{2}{*}{$\begin{array}{l}\text { Gestión de valores } \\
\text { perdidos }\end{array}$} & Definición de perdidos & $\begin{array}{l}\text { Los valores perdidos } \\
\text { definidos por el usuario } \\
\text { se tratan como } \\
\text { perdidos. }\end{array}$ \\
\hline & Casos utilizados & $\begin{array}{l}\text { Las estadísticas se } \\
\text { basan en todos los } \\
\text { casos con datos válidos } \\
\text { para todas las variables } \\
\text { del análisis. }\end{array}$ \\
\hline Sintaxis & & $\begin{array}{l}\text { ROC } \\
\text { MedidaRelTpfQTpost BY } \\
\text { Muertelntrahospitalaria } \\
\text { (1) } \\
\text { IPLOT=CURVE } \\
\text { (REFERENCE) } \\
\text { /PRINT=SE } \\
\text { COORDINATES } \\
\text { /CRITERIA=CUTOFF } \\
\text { (INCLUDE) TESTPOS } \\
\text { (LARGE) DISTRIBUTION } \\
\text { (FREE) CI(95) } \\
\text { /MISSING=EXCLUDE. }\end{array}$ \\
\hline \multirow[t]{2}{*}{ Recursos } & Tiempo de procesador & 00:00:00.24 \\
\hline & Tiempo transcurrido & 00:00:00.00 \\
\hline
\end{tabular}

Figura 4. Curva ROC de la relación Tp-f/QTc posangioplastia para predicción de la mortalidad intrahospitalaria. Área bajo la curva $=0.952 ; \mathrm{p}<0.028$ (Continúa). 


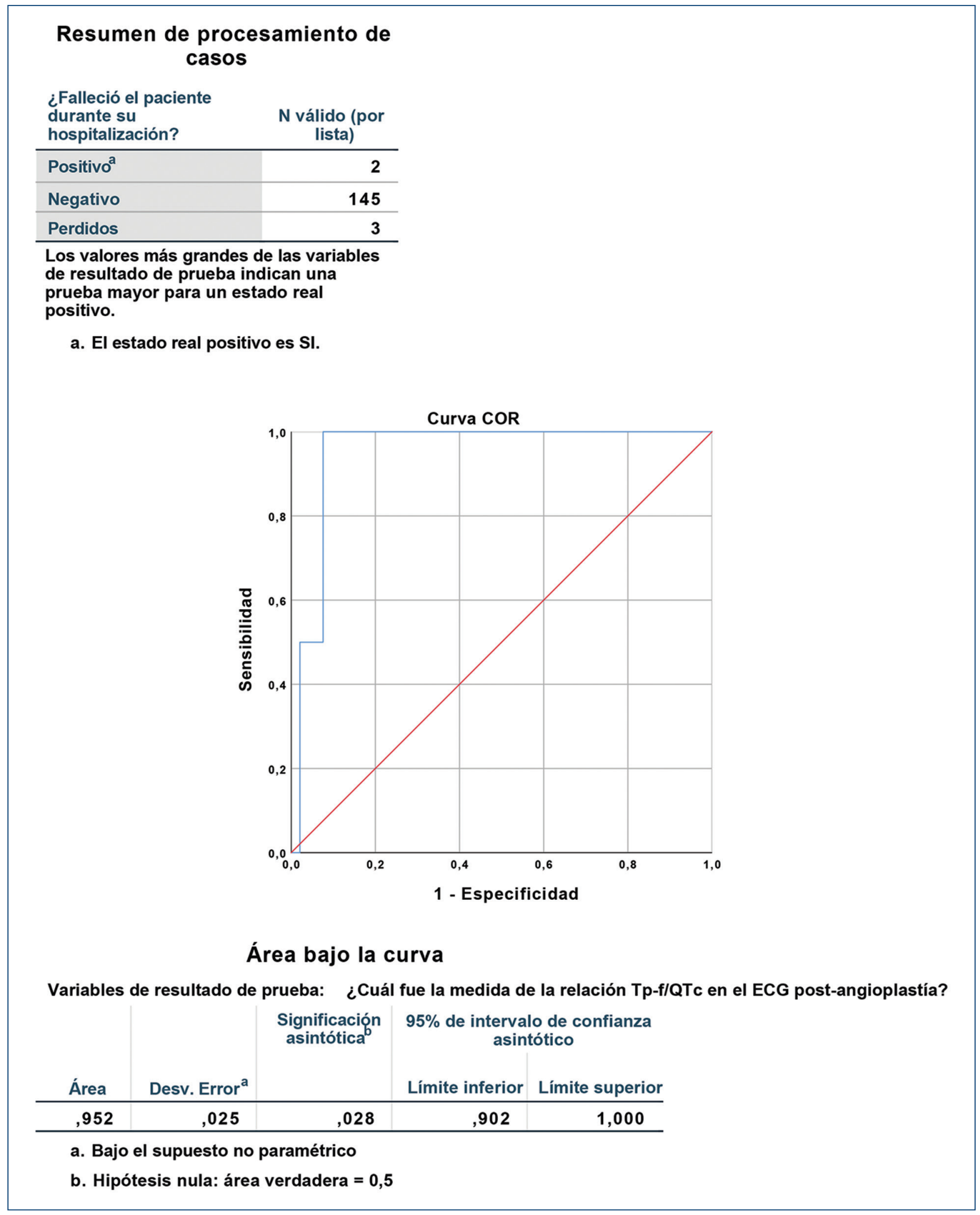

Figura 4. Curva ROC de la relación Tp-f/QTc posangioplastia para predicción de la mortalidad intrahospitalaria. Área bajo la curva $=0.952 ; \mathrm{p}<0.028$ (Continúa). 


\begin{tabular}{|c|c|c|}
\hline \multicolumn{3}{|c|}{ Coordenadas de la curva } \\
\hline \multicolumn{3}{|c|}{ Variables de resultado de prueba: ¿Cuál fue la medida de la relación Tp-f/QTc en el ECG post-angioplastía? } \\
\hline $\begin{array}{l}\text { Positivo si es } \\
\text { mayor o igual } \\
\text { que }\end{array}$ & Sensibilidad & $\begin{array}{c}1- \\
\text { Especificidad }\end{array}$ \\
\hline, 0000 & 1,000 & 1,000 \\
\hline ,1150 & 1,000 & ,993 \\
\hline, 1300 & 1,000 & ,986 \\
\hline, 1450 & 1,000 & ,952 \\
\hline , 1550 & 1,000 & ,938 \\
\hline , 1650 & 1,000 & 897 \\
\hline, 1750 & 1,000 &, 876 \\
\hline , 1850 & 1,000 & ,821 \\
\hline , 1950 & 1,000 & ,759 \\
\hline ,2050 & 1,000 & ,676 \\
\hline ,2150 & 1,000 &, 586 \\
\hline, 2250 & 1,000 &, 545 \\
\hline ,2350 & 1,000 &, 428 \\
\hline, 2450 & 1,000 &, 359 \\
\hline, 2550 & 1,000 & ,303 \\
\hline ,2650 & 1,000 & ,221 \\
\hline ,2750 & 1,000 & ,200 \\
\hline ,2850 & 1,000 & ,166 \\
\hline, 2950 & 1,000 & ,145 \\
\hline, 3050 & 1,000 & ,110 \\
\hline, 3150 & 1,000 &, 097 \\
\hline ,3250 & 1,000 & ,076 \\
\hline, 3350 &, 500 &, 076 \\
\hline, 3500 &, 500 &, 069 \\
\hline, 3650 &, 500 &, 055 \\
\hline, 3800 &, 500 &, 034 \\
\hline, 4050 &, 500 & ,028 \\
\hline, 4450 &, 500 &, 021 \\
\hline, 4750 &, 000 &, 021 \\
\hline, 5100 &, 000 &, 014 \\
\hline, 5450 &, 000 &, 007 \\
\hline 1,0000 &, 000 &, 000 \\
\hline $\begin{array}{l}\text { a. El valor de } \\
\text { corte más } \\
\text { corte son I }\end{array}$ & $\begin{array}{l}\text { corte más pe } \\
\text { grande es el } \\
\text { os promedios }\end{array}$ & $\begin{array}{l}\text { queño es el valor mínimo de prueba observado menos } 1 \text { y el valor de } \\
\text { alor máximo de prueba observado más } 1 \text {. Todos los demás valores de } \\
\text { de los dos valores de prueba observados solicitados consecutivos. }\end{array}$ \\
\hline
\end{tabular}

Figura 4. Curva ROC de la relación Tp-f/QTc posangioplastia para predicción de la mortalidad intrahospitalaria. Área bajo la curva $=0.952 ; \mathrm{p}<0.028$. 
Es importante destacar que el Sistema Tec Salud cuenta con un protocolo bien establecido para la atención de SICA, y este hecho aportó cierta uniformidad respecto al manejo intrahospitalario de los pacientes estudiados. En aquellos pacientes en quienes se realizó angioplastia urgente, la media de los tiempos puerta-aguja y puerta-angioplastia inclusive es menor que la considerada estándar internacionalmente.

Entre sus limitantes se encuentra la naturaleza observacional y retrospectiva del estudio, que confiere la inclusión de estrategias de tratamiento no estandarizadas. Asimismo, el análisis consecutivo de eventos posteriores al SICA inicial se basó en la revisión de registros en expediente electrónico para seguimiento en consulta, sin contar con un protocolo uniforme para la vigilancia de los pacientes ni de su tratamiento posterior al evento primario.

La medición de los intervalos Tp-f y QT se efectuó de forma manual usando ECG convencional de 12 derivaciones a una velocidad de registro de $25 \mathrm{~mm} / \mathrm{s}$, y este método podría haber reducido la precisión de las mediciones.

\section{Conclusiones}

Tras el análisis de los datos se pudo concluir que existe un acortamiento significativo en la relación Tp-f/ QTc tras la angioplastia en los pacientes con SICA. Es importante destacar que solo al establecer un punto de corte $\geq 0.29$ la relación Tp-f/QTc preangioplastia parece guardar relación con la presentación de eventos adversos periprocedimiento de forma global. Una relación Tp-f/QTc $\geq 0.25$ tras la angioplastia tiene una relación estadísticamente significativa con la presentación de eventos cardiovasculares en el primer año tras el evento y la necesidad de reintervención. Una relación Tp-f/QTc $\geq 0.29$ tras la angioplastia tiene una relación estadísticamente significativa con la presentación de muerte posterior a 1 año, la aparición de eventos cardiovasculares en el primer año y la necesidad de reintervención. Esta medida de la DTMRV puede servir como un predictor de muerte intrahospitalaria, eventos cardiovasculares y reintervención a 1 año en pacientes con SICA tratados con angioplastia.

\section{Financiamiento}

Los autores declara no haber recibido ningún tipo de financiamiento para la realización del estudio.

\section{Conflicto de intereses}

Los autores declaran no tener ningún conflicto de intereses.

\section{Responsabilidades éticas}

Protección de personas y animales. Los autores declaran que para esta investigación no se han realizado experimentos en seres humanos ni en animales.

Confidencialidad de los datos. Los autores declaran que han seguido los protocolos de su centro de trabajo sobre la publicación de datos de pacientes.

Derecho a la privacidad y consentimiento informado. Los autores declaran que en este artículo no aparecen datos de pacientes.

\section{Bibliografía}

1. Instituto Nacional de Estadística y Geografía. INEGI. Principales causas de mortalidad por residencia habitual, grupos de edad y sexo del fallecido. (Consultado el 12 de abril de 2018.) Disponible en: http://www.inegi. org.mx/est/contenidos/proyectos/registros/vitales/mortalidad/tabulados/ ConsultaMortalidad.asp.

2. Bayés de Luna A, Coumel P, Leclercq JF. Ambulatory sudden cardiac death: mechanisms of production of fatal arrhythmia on the basis of data from 157 cases. Am Heart J. 1989;117:151-9.

3. Antzelevitch C. Cellular basis for the repolarization waves of the ECG. Ann N Y Acad Sci. 2006;1080:268-81.

4. Antzelevitch C. Tpeax-Tend interval as an index of transmural dispersion of repolarization. Eur J Clin Invest. 2001;31:555-7.

5. Galinier M, Balanescu S, Fourcade J, Dorobantu M, Boveda S, Massabuau $\mathrm{P}$, et al. Prognostic value of ventricular arrhythmias in systemic hypertension. J Hypertens. 1997;15:1779-83.

6. Schwartz PJ, Wolf S. QT interval prolongation as predictor of sudden death in patients with myocardial infarction. Circulation. 1978:57:1074-77.

7. Sundqvist K, Sylvén C. Cardiac repolarization properties during standardized exercise test as studied by QT, QT peak and terminal T-wave intervals. Clin Physiol. 1989:9:419-25.

8. Gupta P, Patel C, Patel H, Narayanaswamy S, Malhotra B, Green JT, et al. Tp-e/QT ratio as an index of arrhytmogenesis. J Electrocardiol. 2008;41:1114-6.

9. Erikssen G, Liestøl K, Gullestad L, Haugaa KH, Bendz B, Amlie JP. The terminal part of the QT interval (T peak to $T$ end): a predictor of mortality after acute myocardial infarction. Ann Noninvasive Electrocardiol. 2012;17:85-94.

10. Hetland M, Haugaa KH, Sarvari SI, Erikssen G, Kongsgaard E, Edvardsen T. A novel ECG-index for prediction of ventricular arrhythmias in patients after myocardial infarction. Ann Noninvasive Electrocardiol. 2014;19:330-7.

11. Zhao X, Xie Z, Chu Y, Yang L, Xu W, Yang X, et al. Association between $\mathrm{Tp}-\mathrm{e} / \mathrm{QT}$ ratio and prognosis in patients undergoing percutaneous coronary intervention for ST-segment elevation myocardial infarction. Clin Cardiol. 2012;35:559-64. 